\title{
Determination of optimal loading and maintenance doses for continuous infusion of vancomycin in critically ill patients: Population pharmacokinetic modelling and simulations for improved dosing schemes
}

\author{
Dinh H. Vu ${ }^{\mathrm{a}, *}$, Duy A. Nguyen ${ }^{\mathrm{a}}$, Isabelle K. Delattre ${ }^{\mathrm{b}}$, Trong T. Ho ${ }^{\mathrm{a}}$, Hong G. Doc, \\ Hong N. Pham ${ }^{\mathrm{d}}$, Xuan C. Dao ${ }^{\mathrm{e}}$, Nhan T. Tran ${ }^{\mathrm{c}}$, Gia B. Nguyen ${ }^{\mathrm{e}}$, Françoise Van Bambeke ${ }^{\mathrm{b}}$, \\ Paul M. Tulkens ${ }^{b}$, Hoang A. Nguyen ${ }^{a}$ \\ a National Drug Information and Adverse Drug Reaction Monitoring Center, Hanoi University of Pharmacy, Hanoi, Vietnam \\ ${ }^{\mathrm{b}}$ Louvain Drug Research Institute, Université catholique de Louvain, Brussels, Belgium \\ ${ }^{\mathrm{c}}$ Department of Pharmacy, Bach Mai Hospital, Hanoi, Vietnam \\ ${ }^{\mathrm{d}}$ Department of Microbiology, Bach Mai Hospital, Hanoi, Vietnam \\ e Intensive Care Unit, Bach Mai Hospital, Hanoi, Vietnam
}

\section{A R T I C L E I N F O}

\section{Article history:}

Received 1 June 2019

Accepted 28 September 2019

Editor: H. Derendorf

Keywords:

Vancomycin

Continuous infusion

Loading dose

Maintenance dose

Critically ill

\begin{abstract}
A B S T R A C T
Objectives: Despite extensive clinical use, limited data are available on optimal loading and maintenance doses of vancomycin in critically ill patients. This study aimed to develop a rational approach for optimised dosage of vancomycin given in a continuous infusion in critically ill patients.

Methods: Vancomycin pharmacokinetic (PK) data (total serum concentrations) were obtained from 55 intensive care unit (ICU) patients (Bach Mai Hospital, Hanoi, Vietnam) receiving a $20 \mathrm{mg} / \mathrm{kg}$ loading dose followed by continuous infusion stratified by creatinine clearance (CLCr). Population PK modelling and Monte Carlo simulations were performed using a nonlinear mixed-effects modelling (NONMEM) program for a target of $20-30 \mathrm{mg} / \mathrm{L}$ to optimise efficacy and minimise nephrotoxicity.

Results: A two-compartment model with first-order elimination best fitted the PK data with central and peripheral volumes of distribution of 1.01 and $2.39 \mathrm{~L} / \mathrm{kg}$, respectively (allometric scaling to a $70 \mathrm{~kg}$ standard subject). The population total clearance of $3.63 \mathrm{~L} / \mathrm{h}$ was only explained by renal function in the covariate and final model. The simulations showed that a $25-\mathrm{mg} / \mathrm{kg}$ loading dose infused over 90 minutes was optimal to reach the target range. The optimal maintenance dose for low renal function $(\mathrm{CLCr}<45 \mathrm{~mL} / \mathrm{min})$ was $1000-1500 \mathrm{mg} /$ day. For augmented renal clearance $(\mathrm{CLCr}>130 \mathrm{~mL} / \mathrm{min})$ the dose should be up to $3500 \mathrm{mg} / \mathrm{day}$ or even $4500 \mathrm{mg} /$ day to achieve adequate exposure. These simulated maintenance doses were larger than previously proposed for non-ICU patients.

Conclusion: Large loading and maintenance doses of vancomycin are generally needed in critically ill patients. Because of high interindividual variability in vancomycin PK, drug monitoring may still be necessary.
\end{abstract}

(C) 2019 Elsevier B.V. and International Society of Chemotherapy. All rights reserved.

\section{Introduction}

Despite the approval of several new anti-Gram-positive agents, vancomycin remains a mainstay treatment for infection caused by

\footnotetext{
* Corresponding author: National Drug Information and Adverse Drug Reaction Monitoring Center, Hanoi University of Pharmacy, 13-15, Le Thanh Tong, Hoan Kiem, Hanoi, Vietnam. Tel.: +84 904250745.

E-mail address: vudinhhoa@gmail.com (D.H. Vu).
}

methicillin-resistant Staphylococcus aureus (S. aureus) (MRSA) or Enterococci in hospitalised patients; however, it requires careful and continuing dosage adjustments [1-4]. The $\mathrm{AUC}_{0-24} / \mathrm{MIC}$ (ratio of the area under the 24-h drug concentration-time curve to the minimal inhibitory concentration of vancomycin against the presumed bacterial pathogen) of vancomycin best predicts optimal microbiological response and favourable clinical outcome [5-8]. Yet, variations of its distribution and elimination parameters in critically ill patients [9-11] often calls for substantial changes 
in both initial and maintenance dosing. While vancomycin was originally recommended for administration by intermittent infusion (II), several studies have shown that continuous infusion (CI) is as effective [12], while greatly facilitating therapeutic drug monitoring (TDM) [11,13]. In addition, while high peak levels of vancomycin are unnecessary, controlling its steady state plateau to a sufficiently low level can subside nephrotoxicity [7,12,14]. However, initiation of a vancomycin $\mathrm{CI}$ calls for a loading dose that quickly reaches blood levels of $20-30 \mathrm{mg} / \mathrm{L}[11,15]$ to cover isolates with vancomycin MICs of 1-2 mg/L. Thus, loading doses of $15-20 \mathrm{mg} / \mathrm{kg}$ are often recommended [11,12] but may frequently lead to subtherapeutic concentrations [15-18]. A simulation study using a one-compartment model applied to critically ill patients showed that a loading dose of $35 \mathrm{mg} / \mathrm{kg}$ could be necessary [15], but also resulted in a large proportion of patients with supratherapeutic vancomycin concentrations [16]. Conversely, patients with augmented renal clearance (ARC) could experience suboptimal exposure [16-18]. This has triggered population pharmacokinetic (PK) modelling and simulation studies to identify which loading and maintenance vancomycin doses could be optimal in critically ill patients. The current study observed that a larger loading dose than often proposed in current practice is necessary, as well as a large maintenance dose in cases of ARC. Yet, TDM remains essential to ensure optimised therapy ${ }^{1}$.

\section{Methods}

\subsection{Patient population}

This observational study prospectively collected information from medical records of patients admitted to the intensive care unit (ICU) of the Bach Mai hospital, Hanoi, Vietnam, and suffering from (or suspected of) an infection for which vancomycin was indicated. The decision to use $\mathrm{CI}$, initial dosing (loading dose), and further dose adjustment (during $\mathrm{CI}$ ) were according to the internally approved hospital protocol and based on an earlier publication [8]. Excluded patients were those with: (i) age $<18$ years; (ii) pregnancy or lactation; (iii) previous use of vancomycin II within 48 hours before initiating CI; (iv) CI of vancomycin for $<24$ hours; and $(v)$ renal replacement therapy or extracorporeal membrane oxygenation. Key characteristics of all enrolled patients (Table 1) were recorded.

\subsection{Therapeutic drug monitoring program}

Vancomycin was assayed in plasma by homogenous enzyme immunoassay (Cobas c 501 systems, Hoffman-La Roche, Basel Switzerland; analytical range: $1.7-80 \mathrm{mg} / \mathrm{L}$ ). Most treatments with vancomycin were started empirically with a $20 \mathrm{mg} / \mathrm{kg}$ loading dose (over 60-120 minutes). The maintenance dose was then set using calculated (Cockcroft-Gault) creatinine clearance (CLCr) and following a previously published algorithm (Supplementary Material in reference [8]). A first vancomycin level was obtained within 12-24 hours and the infusion rate was increased or combined with a second loading dose if this level was $<20 \mathrm{mg} / \mathrm{L}$ or decreased or discontinued for 6 hours before resuming with a lower infusion rate if $>30 \mathrm{mg} / \mathrm{L}$ [8]. Additional levels were obtained over the next days with corrections of the infusion rate made until the targeted concentration range (20-30 mg/L) was obtained, after which samples were collected on the next 3 days to check for level variations.

\footnotetext{
${ }^{1}$ A preliminary account of these findings was presented at the 29th European Congress of Clinical Microbiology and Infection (29th ECCMID; presentation 01160 - Session 'PK/PD to guide dosing in special populations' - 16/04/2019 available for download from http://www.facm.ucl.ac.be/posters/2019/29th-ECCMID/ Vu-et-al-oral-01160-ECCMID2019.pdf).
}

Table 1

Patient characteristics $(\mathrm{n}=55)$.

\begin{tabular}{|c|c|}
\hline Characteristics & Values \\
\hline \multicolumn{2}{|l|}{ Demographic data } \\
\hline Sex (male), $\mathrm{n}(\%)$ & $36(65.5)$ \\
\hline Age (years) & $55 \pm 18$ \\
\hline Actual body weight $(\mathrm{kg})$ & $55.9 \pm 11.1$ \\
\hline \multicolumn{2}{|c|}{ Clinical characteristics (when vancomycin was started) } \\
\hline APACHE II score & $14[8-19]$ \\
\hline SOFA score & $4[3-6]$ \\
\hline Charlson comorbidity index & $1[1-3]$ \\
\hline Baseline Clcr (mL/min) & $76.5 \pm 36.4$ \\
\hline Mechanical ventilation, n (\%) & $36(65.5)$ \\
\hline Vasopressor, n (\%) & $6(10.9)$ \\
\hline Septic shock, n (\%) & $4(7.3)$ \\
\hline \multicolumn{2}{|l|}{ Concomitant use of nephrotoxic agents, $n(\%)$} \\
\hline Furosemide & $31(56.4)$ \\
\hline Non-steroid anti-inflammatory drugs & $9(16.4)$ \\
\hline Renin-angiotensin-aldosterone system inhibitors & $5(9.1)$ \\
\hline Amphotericin B & $1(1.8)$ \\
\hline Contrast medias & $1(1.8)$ \\
\hline \multicolumn{2}{|l|}{ Vancomycin usage/sampling } \\
\hline Loading dose $(\mathrm{mg} / \mathrm{kg})$ & $19.7 \pm 2.3$ \\
\hline Daily maintenance dose (mg) & $2261 \pm 1052$ \\
\hline Vancomycin treatment duration (days) & $6[5-11]$ \\
\hline Blood samples per patient & $4[3-6]$ \\
\hline \multicolumn{2}{|l|}{ MIC isolated pathogens $(n=7)\left({ }^{*}\right)$} \\
\hline MIC $0.5 \mathrm{mg} / \mathrm{L}$ & $2(28.6)$ \\
\hline MIC $0.75 \mathrm{mg} / \mathrm{L}$ & $2(28.6)$ \\
\hline MIC $1 \mathrm{mg} / \mathrm{L}$ & $3(42.8)$ \\
\hline
\end{tabular}

Data are presented in $\mathrm{n}(\%)$ for binominal variables, mean \pm standard deviation for normal distribution or median [interquartile range] for non-normal distribution variables

MIC: minimum inhibitory concentration; (*): Staphylococcus aureus (5) and Enterococcus spp. (2)

Clcr: clearance creatinine estimated from Cockcroft-Gault equation

\subsection{MIC determinations}

S. aureus and Enterococcus isolates from the collected specimens were stored at $-70^{\circ} \mathrm{C}$ before testing and MICs were determined with vancomycin MIC Test Strips (Liofilchem S.r.L., Roseto degli Abruzzi, Italy) using Mueller-Hinton agar medium (BD [Becton, Dickinson and Co.], Franklin Lakes, NJ, USA) with incubation under ambient air at $35 \pm 2{ }^{\circ} \mathrm{C}$ (https://clsi.org/).

\subsection{Population pharmacokinetic modelling}

Population PK analysis was carried out using the nonlinear mixed-effects modelling program (NONMEM Version VI) (double precision; ICON Development Solutions, LLC, Ellicott City, MD), with G77 Fortran for compilation and execution, and with Perlspeaks-NONMEM (PsN) tool kit and Xpose (Version 4) for statistical and graphic model evaluation $[19,20]$.

\subsubsection{Population parameter estimation}

The population parameters of vancomycin were assessed using the first-order conditional estimation with interaction method. Both one-compartment and two-compartment models with firstorder elimination were tested to describe the concentration-time data of vancomycin. The PK analyses were parameterised using volume(s) of distribution and clearance(s). The interindividual variability $(\eta)$ in PK parameters was described by an exponential model:

$\mathrm{P}_{\mathrm{i}}=\mathrm{P}_{\text {pop }} \bullet \exp \left(\eta_{\mathrm{i}}\right)$

where $\mathrm{P}_{\mathrm{i}}$ is the value of the $\mathrm{PK}$ parameter $\mathrm{P}$ in the ith individual, $\mathrm{P}_{\text {pop }}$ is the population estimation of $\mathrm{P}$, and $\eta_{\mathrm{i}}$ quantifies the deviation of $\mathrm{P}_{\mathrm{i}}$ from $\mathrm{P}_{\mathrm{pop}} ; \eta$ is assumed to be a normal random variable, with a mean of 0 and variance of $\omega^{2}$. 
An additive, proportional, or a combined proportional and additive error model was used to describe the residual unexplained variability $(\varepsilon)$ :

$\mathrm{Y}=\mathrm{IPRED}+\varepsilon_{\mathrm{add}}$

$\mathrm{Y}=\operatorname{IPRED} \bullet\left(1+\varepsilon_{\text {prop }}\right)$

$\mathrm{Y}=\operatorname{IPRED} \bullet\left(1+\varepsilon_{\text {prop }}\right)+\varepsilon_{\text {add }}$

where $\mathrm{Y}$ is the observed concentration, IPRED is the individual predicted concentration without residual error, and $\varepsilon_{\text {prop }}$ (proportional component) and $\varepsilon_{\text {add }}$ (additive component) quantify the deviation of $\mathrm{Y}$ from IPRED; $\varepsilon$ is assumed to be a normal random variable, with a mean of 0 and a variance of $\sigma^{2}$. To enable further comparison with results from other patient populations and make the conclusions normative, an allometric weight model was applied for scaling PK parameter values to a standard body weight of $70 \mathrm{~kg}$ [21,22]:

$\mathrm{P}_{\mathrm{i}}=\mathrm{P}_{\text {std }} \bullet\left(\frac{\mathrm{WT}_{\mathrm{i}}}{\mathrm{WT}_{\text {std }}}\right)^{\mathrm{PWR}}$

where $P_{i}$ is the PK parameter in the ith individual, $W T_{i}$ is the weight in the ith individual, $P_{\text {std }}$ is the weight standardised value of the PK parameter in an individual with a standard weight $\left(\mathrm{WT}_{\text {std }}\right)$ of $70 \mathrm{~kg}$; PWR exponent was 1 for volume of distribution(s) and 0.75 for clearance(s) [23].

Competing models were evaluated using the NONMEM objective function value (OFV), precision of estimates, and basic goodness-of-fit plots (i.e. observed vs. predicted concentrations, conditional weighted residuals vs. predicted concentrations and vs. time after dose) [24]. Parameter uncertainty was expressed as the relative standard error of estimates (RSE).

The following patient-specific covariates were tested for influence on PK parameter estimates: sex; age; renal function; Acute Physiology, Age, Chronic Health Evaluation II (APACHE II) score; Sequential Organ Failure Assessment (SOFA) score; and ventilation. Renal function was assessed from the Cockcroft-Gault equation, using a standard creatinine clearance of $100 \mathrm{~mL} / \mathrm{min}$ for a nominal 70-kg subject:

$\mathrm{RF}=\frac{\mathrm{CLCr}_{\mathrm{i}}}{\mathrm{CLCr}_{\text {std }}}$

$\mathrm{CLCr}_{\mathrm{i}}=\frac{(140-\text { age }) \bullet \mathrm{WT}_{\text {std }}}{\mathrm{SCr} \bullet 72} \bullet(0.85$ if female $)$

where $\mathrm{CLCr}_{\mathrm{i}}$ is the creatinine clearance of the ith individual, $\mathrm{CLCr}_{\text {std }}$ is the standard creatinine clearance of $100 \mathrm{~mL} / \mathrm{min}, \mathrm{WT}_{\text {std }}$ is the standard weight of $70 \mathrm{~kg}$, and $\mathrm{SCr}$ is the serum creatinine $(\mathrm{mg} / \mathrm{dL})$.

Individual Bayes estimates of PK parameters were generated, and covariate-PK parameter relationships were visually inspected and investigated in NONMEM. The final model was built using a two-stage approach:

i. In the first step, covariates were separately added to the structural model using a power function. A decrease in OFV $\geq 6.64$ ( $\chi^{2}$ distribution, $P \leq 0.01$, degree of freedom $=1$ ) from the structural model was considered statistically significant.

ii. In the second step, a full model was built, including all covariates that showed significant influence on PK parameters, from which a backward selection was performed. Covariates which upon deletion resulted in an increase in OFV $\geq 10.83\left(\chi^{2}\right.$ distribution, $P \leq 0.001$, degree of freedom $=1$ ) were retained in the final model.

\subsubsection{Model evaluation}

To estimate the uncertainty in population parameters, a nonparametric bootstrap procedure was performed for the developed model (1000 replicates/model) [20]. Predictive performance of population PK models was assessed using visual predictive check [25].

\section{Simulations}

Monte-Carlo simulations based on the final population model were performed in two steps. This approach enabled the loading dose to be optimised in the first step and the maintenance dose in the second step, which should have enabled a dosing scheme to be proposed that would allow optimal blood levels to be obtained during the whole treatment period. First, simulations were carried out to find the optimal loading dose for 1000 patients receiving (i) 10 and $15 \mathrm{mg} / \mathrm{kg}$ infused in 1 hour; (ii) 20 and $25 \mathrm{mg} / \mathrm{kg}$ infused in $1.5 \mathrm{~h}$ or $2 \mathrm{~h}$, respectively; and (iii) 30,35 or $40 \mathrm{mg} / \mathrm{kg}$ infused in $3 \mathrm{~h}$ to select which regimen yielded the highest probability of reaching the target concentration range. Second simulations were performed with the optimal loading doses in previous steps followed by a daily maintenance dose ranging from $300-4500 \mathrm{mg}$, which was stratified by the $\mathrm{CLCr}$ of the patients. The $\mathrm{CLCr}$ was categorised to $<10,10-20,21-30,31-45,46-60,61-85,86-110$, $111-130,131-180$, and $181-240 \mathrm{~mL} / \mathrm{min}$. The proportion of 1000 simulated patients with concentrations at $24 \mathrm{~h}$ post dose attaining the targeted concentration range were assessed to find the optimal maintenance dose. The simulated results were visualised using ggplot2 (https://cran.r-project.org/web/packages/ggplot2/index. html) package on R3.5.2.

\section{Results}

The key characteristics of the 55 eligible patients are shown in Table 1. Of note: (i) baseline CLCr was $76.5 \pm 36.4 \mathrm{~mL} / \mathrm{min}$; (ii) the average vancomycin loading dose was $19.7 \pm 2.3 \mathrm{mg} / \mathrm{kg}$ followed by an average daily maintenance dose of $2261 \pm 1052 \mathrm{mg}$ for a median duration of 6 days [IQR: 5-11]; (iii) co-administration of one or more high-risk nephrotoxicity agents was frequent; (iv) MICs obtained for seven organisms (five S. aureus; two enterococci) ranged from $0.5-1 \mathrm{mg} / \mathrm{L}$.

Two hundred and seventy-four blood samples were collected and available for TDM. A two-compartment structure model with zero-order input and first-order elimination best fitted the vancomycin data and was therefore selected. The model was parameterised in terms of central volume of distribution (V1), peripheral volume of distribution (V2), inter-compartmental clearance $(\mathrm{Q})$, and total body clearance (CL). A proportional error model adequately described the residual variability. The final population parameter estimates with their respective RSE are listed in Table 2. The estimated V1 and CL were $1.01 \mathrm{~L} / \mathrm{kg}$ and $3.63 \mathrm{~L} / \mathrm{h}$, respectively. The interindividual variability of these parameters was $30.2 \%$ and $53.1 \%$, respectively. Only renal function could be retained in the full and final model as a covariate to predict the CL of vancomycin. The results of 1000 bootstrap replicates presented as medians, with 2.5th and 97.5th percentiles of each PK parameter showing marginal differences to the estimation from the model. Basic goodness-of-fit plots are displayed in Figure 1 (no significant bias observed). The visual predictive check plot suggested that $96.7 \%$ observed concentrations fell within the 95\% prediction interval $(0 \%$ observed concentrations were outside the lower limit, and $3.3 \%$ observed concentrations were outside the upper limit of the 95\% prediction interval) (Figure 2 ).

A loading dose $\leq 15 \mathrm{mg} / \mathrm{kg}$ resulted in an important risk of vancomycin concentrations $<20 \mathrm{mg} / \mathrm{L}$. With a loading dose of 20 $\mathrm{mg} / \mathrm{kg}$, about two-thirds of patients did not achieve the targeted 
Table 2

Final population estimates of vancomycin in critically ill patients, and bootstrap validation.

\begin{tabular}{|c|c|c|c|c|c|}
\hline \multirow[t]{2}{*}{ Parameter } & \multirow[t]{2}{*}{ Unit } & \multicolumn{2}{|c|}{ Final model } & \multicolumn{2}{|c|}{ Bootstrap $(n=1000)$} \\
\hline & & estimate & (RSE) & Median & (2.5-97.5th percentile) \\
\hline \multicolumn{6}{|c|}{ Pharmacokinetic parameter } \\
\hline V1 & $\mathrm{L} / \mathrm{kg}$ & 1.01 & $(15.0 \%)$ & 1.11 & $(0.80-1.40)$ \\
\hline V2 & $\mathrm{L} / \mathrm{kg}$ & 2.39 & $(23.2 \%)$ & 2.61 & $(1.26-13.6)$ \\
\hline Q & $\mathrm{L} / \mathrm{h}$ & 1.92 & $(26.6 \%)$ & 1.90 & $(0.96-3.41)$ \\
\hline $\mathrm{CL}$ & $\mathrm{L} / \mathrm{h}$ & 3.63 & $(10.8 \%)$ & 3.51 & $(2.14-4.33)$ \\
\hline \multicolumn{6}{|l|}{ Covariate } \\
\hline $\mathrm{P}_{\mathrm{CL}-\mathrm{RF}}$ & & 1.01 & $(18.3 \%)$ & 1.06 & $(0.65-1.97)$ \\
\hline \multicolumn{6}{|c|}{ Interindividual variability } \\
\hline V1 (CV) & $\%$ & 30.2 & $(41.2 \%)$ & 27.6 & $(8.09-47.6)$ \\
\hline V2 (CV) & $\%$ & 62.0 & $(56.6 \%)$ & 65.0 & $(17.8-203)$ \\
\hline $\mathrm{Q}(\mathrm{CV})$ & $\%$ & 107 & (38.2\%) & 104 & $(36.3-153)$ \\
\hline $\mathrm{CL}(\mathrm{CV})$ & $\%$ & 53.1 & (48.9\%) & 50.9 & $(28.7-80.8)$ \\
\hline \multicolumn{6}{|c|}{ Residual variability } \\
\hline$\varepsilon_{\text {prop }}(\mathrm{CV})$ & $\%$ & 41.4 & $(8.25 \%)$ & 41.4 & $(38.3-45.3)$ \\
\hline OFV & & 1250 & & 1239 & \\
\hline
\end{tabular}

Abbreviations: RSE, relative standard error of estimates; pc, percentile; V1, central volume of distribution; V2, peripheral volume of distribution; Q inter-compartmental clearance; CL, total body clearance; $\mathrm{P}_{\mathrm{CL}-\mathrm{RRT}}$, fractional change on $\mathrm{CL}$ due to renal function (RF); CV, coefficient of variation; $\mathrm{SD}$, standard deviation; OFV, objective function value.

a

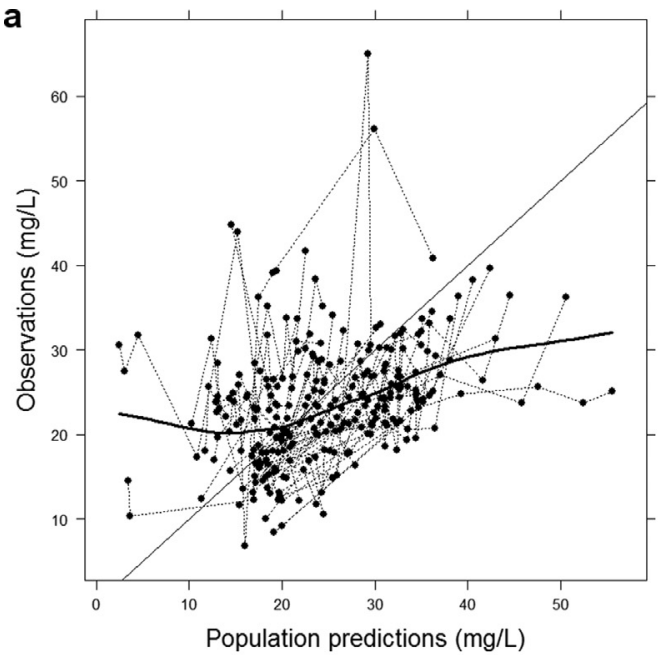

C

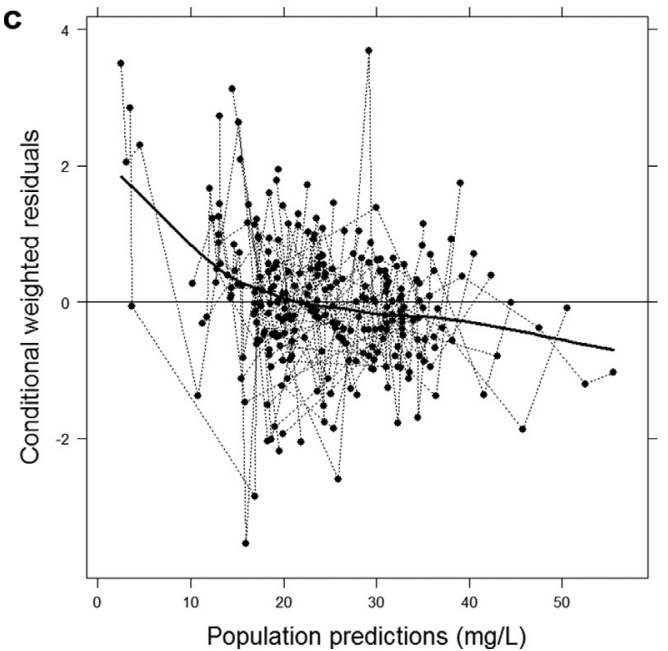

b

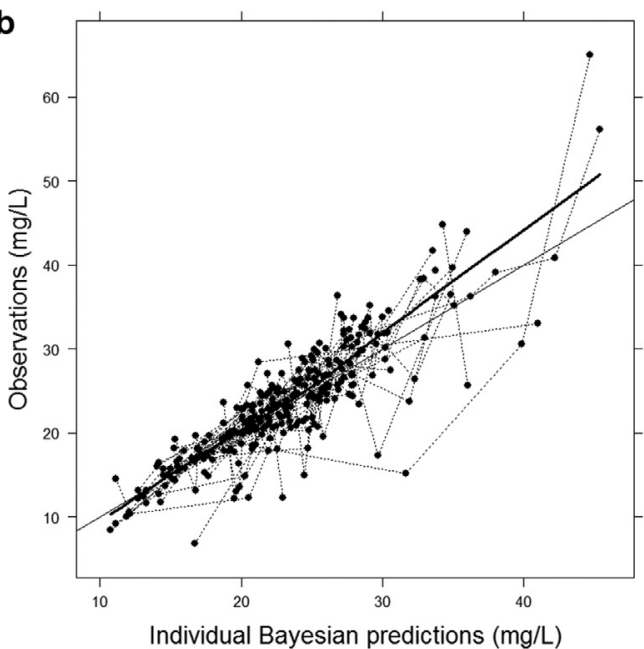

d

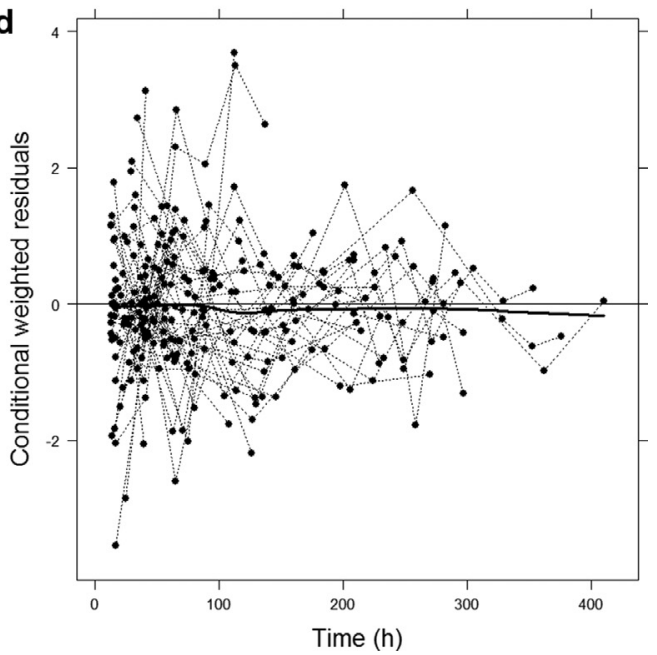

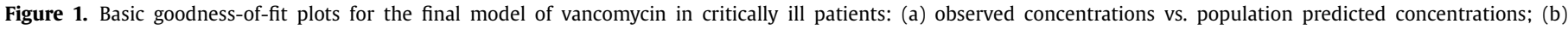

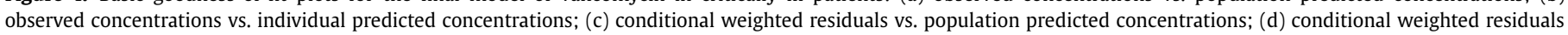
vs. time. The $\mathrm{x}=\mathrm{y}$ line is the identity line. The bold line is the LOESS smooth. The data points of each individual patient are joined by thin lines. 


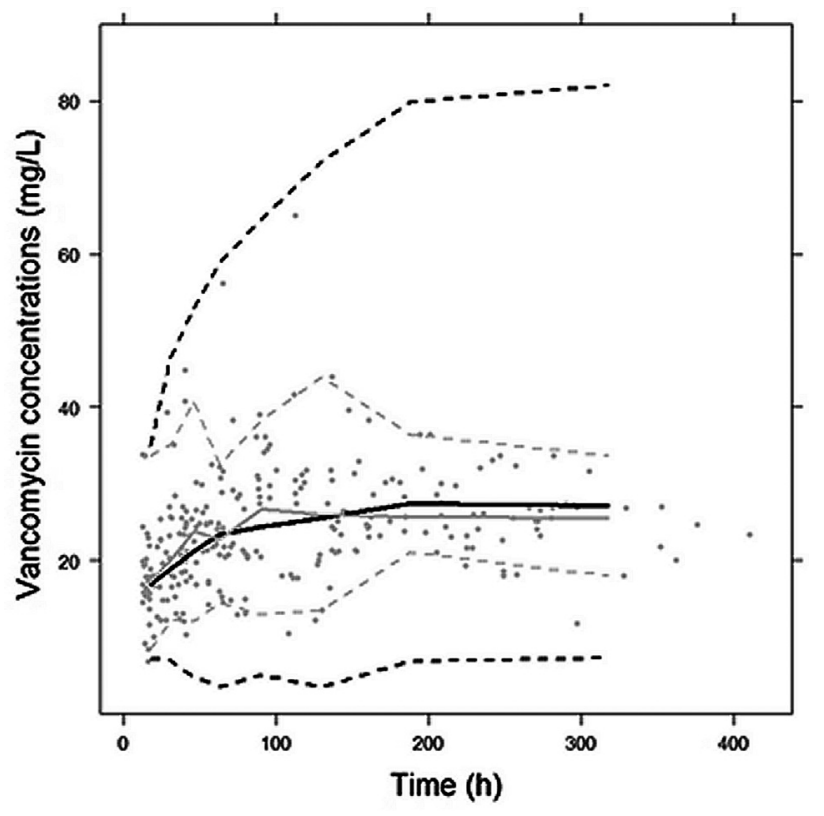

Figure 2. Visual predictive check plot based on 1000 critically ill patients, simulated from the final population estimates. The dashed grey lines present the 2.5 th and 97.5th percentiles of observed data, and the solid grey line depicts the median of observed data. The dashed black lines present the 2.5th and 97.5th percentiles (denoting the $95 \%$ prediction interval), and the solid black line depicts the median of simulated data. The grey dots are the observed concentrations.

concentration range. Increasing the loading dose to $25 \mathrm{mg} / \mathrm{kg}$ or $30 \mathrm{mg} / \mathrm{kg}$ would give patients the highest probability to reach target, but a further increase would result in risk of concentrations > $30 \mathrm{mg} / \mathrm{L}$ (Figure 3).

A daily maintenance dose of $2000-3000 \mathrm{mg}$ for patients with CLCr from $61-130 \mathrm{~mL} / \mathrm{min}$ was expected to yield vancomycin stable serum levels within the desired target range, as it did for nonICU patients [8] since this level is only dependent on vancomycin clearance and not its distribution volume. However, for patients with very low and or very high CLCr, the daily maintenance dose in the current TDM protocol appeared to be inadequate. Thus, the simulation suggested that the daily maintenance dose should be
$1000 \mathrm{mg} /$ day or $1500 \mathrm{mg} /$ day in patients with CLCr $\leq 20$ or $21-45$ $\mathrm{mL} / \mathrm{min}$, respectively. Conversely, the simulation showed that the daily maintenance dose for patients with $\operatorname{ARC}(\mathrm{ClCr}>130 \mathrm{~mL} / \mathrm{min})$ would need to be increased (to $3500 \mathrm{mg} /$ day for CLCr of $130-180$ $\mathrm{mL} / \mathrm{min}$, and even to $4500 \mathrm{mg} /$ day for $\mathrm{CLCr}>181 \mathrm{~mL} / \mathrm{min}$ ) to ensure that blood concentrations are reached within the desired target range (Table 3 ).

\section{Discussion}

The present study showed that the PK of vancomycin $\mathrm{CI}$ is properly described in critically ill patients by a two-compartment model in which CLCr is the significant covariate explaining part of the interindividual variability in the total body clearance of this antibiotic. Using the final model for simulations, it identified a loading dose of $25-30 \mathrm{mg} / \mathrm{kg}$ as being optimal for attaining serum target concentrations of $20-30 \mathrm{mg} / \mathrm{L}$, while higher doses resulted in unnecessarily high concentrations. For vancomycin, reaching plasma levels within a $20-30 \mathrm{mg} / \mathrm{L}$ range guarantees an $\mathrm{AUC}_{0-24}$ allowing coverage of organism(s) with an MIC of $1.2-1.8 \mathrm{mg} / \mathrm{L}$ [6], which is reasonably in line with its current EUCAST S breakpoint $(\leq 2 \mathrm{mg} / \mathrm{L})$ and is appropriate for the strains isolated in this study (Table 1) and observed in the current hospital during the three preceding years (see Supplementary Material Figure S1). This could also minimise the risk of emergence of isolates with reduced susceptibility [26,27]. However, high interindividual variations in vancomycin PK parameters make TDM still mandatory, as advocated earlier [8], and patients with ARC require higher maintenance doses.

The first critical observation is that critically ill patients showed a larger vancomycin distribution volume $(1.01 \mathrm{~L} / \mathrm{kg})$ than previously estimated for non-ICU patients $(0.7 \mathrm{~L} / \mathrm{kg}$ [8]) and as implemented in the current standard protocol and, therefore, required a higher initial loading dose. Thus, the simulation revealed that the loading dose of $15-20 \mathrm{mg} / \mathrm{kg}$ was insufficient to reach the target serum concentration range [15-18]. This was expected for a globally hydrophilic drug (vancomycin $\log \mathrm{D}_{\mathrm{pH} 7}$ is ca. -2 ; https://disco.chemaxon.com/apps/demos/logd/) and has been considered in a previous study [9]. Thus, using a one-compartment model [15,28], Roberts et al. actually reported a distribution volume of $1.5 \mathrm{~L} / \mathrm{kg}$, requiring a loading dose of $35 \mathrm{mg} / \mathrm{kg}$ to fill it to a concentration of $25 \mathrm{mg} / \mathrm{L}$ [15]. However, this resulted in

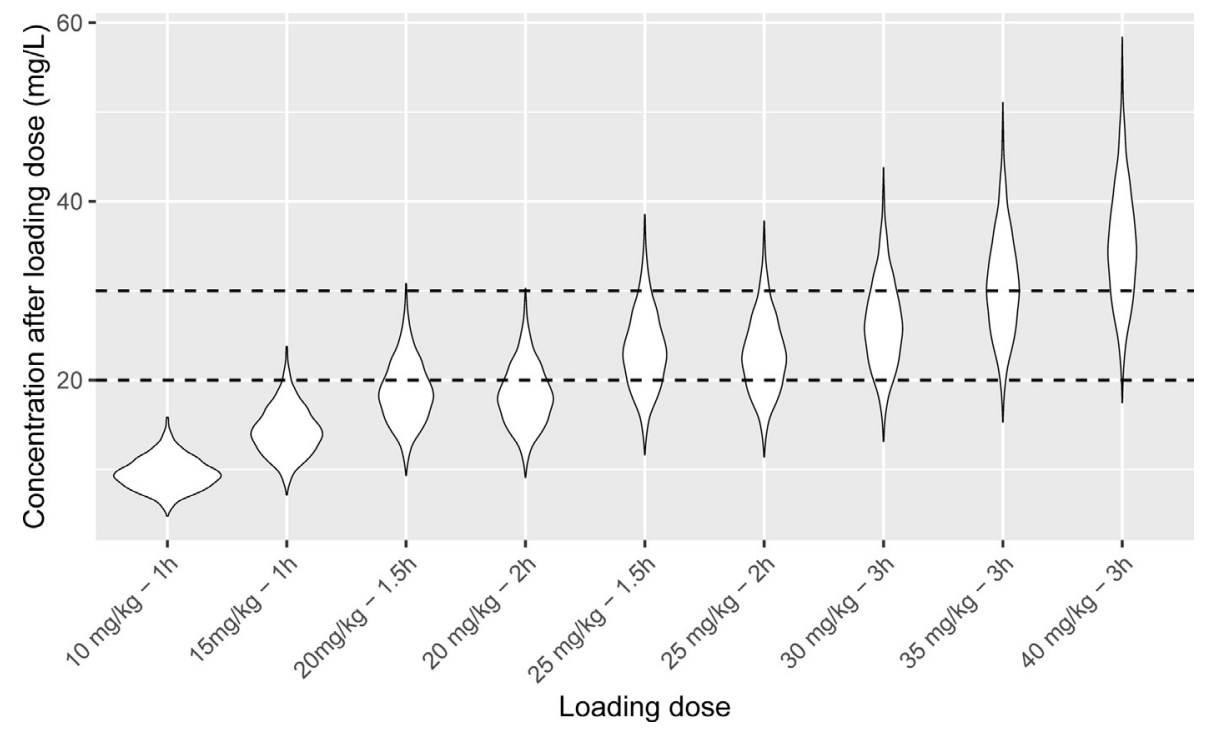

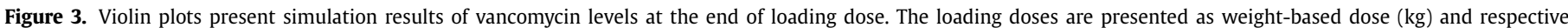
infusion time (hours). The dashed lines present the upper and lower limit of targeted concentration. 
Table 3

Percentage of simulated patient obtaining target level (20-30 mg/L) at 24 hours after a loading dose of $25 \mathrm{mg} / \mathrm{kg}$ infused over 2 hours followed by a maintenance dose.

\begin{tabular}{|c|c|c|c|c|c|c|c|c|c|c|c|}
\hline \multirow{2}{*}{$\begin{array}{l}\mathrm{CLCr} \\
(\mathrm{mL} / \mathrm{min})\end{array}$} & \multicolumn{11}{|c|}{ Maintenance dose (mg/day) } \\
\hline & 300 & 500 & 750 & 1000 & 1500 & 2000 & 2500 & 3000 & 3500 & 4000 & 4500 \\
\hline$<10$ & $16.0 \%$ & $41.2 \%$ & $69.0 \%$ & $74.1 \%$ & $37.5 \%$ & $11.3 \%$ & & & & & \\
\hline $10-20$ & $4.7 \%$ & $22.4 \%$ & $54.9 \%$ & $74.8 \%$ & $54.5 \%$ & $19.4 \%$ & $4.4 \%$ & & & & \\
\hline $21-30$ & & $8.2 \%$ & $34.5 \%$ & $64.9 \%$ & $69.1 \%$ & $31.3 \%$ & $8.9 \%$ & $2.5 \%$ & & & \\
\hline $31-45$ & & & $13.3 \%$ & $41.2 \%$ & $77.4 \%$ & $50.6 \%$ & $19.4 \%$ & $4.9 \%$ & $1.3 \%$ & & \\
\hline $46-60$ & & & & $17.0 \%$ & $68.5 \%$ & $71.5 \%$ & $36.9 \%$ & $13.2 \%$ & $3.3 \%$ & $0.9 \%$ & \\
\hline $61-85$ & & & & & $38.4 \%$ & $77.0 \%$ & $65.6 \%$ & $32.7 \%$ & $13.2 \%$ & $3.9 \%$ & \\
\hline $86-110$ & & & & & & $51.1 \%$ & $79.0 \%$ & $65.3 \%$ & $35.1 \%$ & $14.7 \%$ & $5.0 \%$ \\
\hline $111-130$ & & & & & & & $63.5 \%$ & $81.2 \%$ & $63.1 \%$ & $35.1 \%$ & $15.2 \%$ \\
\hline $131-180$ & & & & & & & $21.8 \%$ & $59.1 \%$ & $77.4 \%$ & $71.0 \%$ & $48.1 \%$ \\
\hline$>181$ & & & & & & & $0.9 \%$ & $9.7 \%$ & $37.3 \%$ & $66.6 \%$ & $79.1 \%$ \\
\hline
\end{tabular}

Abbreviation: CLCr, creatinine clearance.

Figures in bold: largest percentage of patients achieving the target range with the proposed maintenance dose.

Figures in italic: actual percentage of patients that would achieve the target range if using the doses proposed for non-ICU patients [8].

a high median drug level $(44 \mathrm{mg} / \mathrm{L}$ ), creating a definite risk of toxicity if applied blindly to the whole population [16]. Using a one-compartment model while the actual vancomycin plasma level has been reported to follow two-compartment or threecompartment kinetics [29] may lead to an overestimation of the central compartment volume on which the loading dose should be computed. Thus, the two-compartment model used here (as it best fitted the data) probably yields a more realistic estimation of the true central distribution volume. With the proposed loading dose of $25-30 \mathrm{mg} / \mathrm{kg}$, approximately two-thirds of the patients would attain the desired plasma target range. However, because of the interindividual variability observed in the PK model, perfect attainment would not be reached and TDM-based adjustments would be necessary. Indeed, blind increases in the loading dose would create a risk of supratherapeutic levels, as in the validation study mentioned above [16]. Of note, due to its relatively long half-life, modifying the infusion rate of the loading dose will only have a marginal effect on the initial peak concentration of vancomycin. The only significant covariate retained in the final model was renal function, with no significant effect of mechanical ventilation on the estimated PK parameters (contrary to what was reported in a previous study of similar patient sample size [28]).

The second key observation is that a daily maintenance dose of 2000-3000 mg was sufficient for patients with a CLCr of 61-130 $\mathrm{mL} / \mathrm{min}$, indicating that the dosing regimen used in the current TDM program was already optimal for these patients. As previously explained [8], defining the daily maintenance dose needed to maintain the plasma concentration at a desired steadystate level during $\mathrm{CI}$ depends only on drug clearance and is therefore independent of the volume of distribution. This is why the daily maintenance dose was expressed in $\mathrm{mg}$ and not, as often erroneously presented by others, in $\mathrm{mg} / \mathrm{kg}$. This prevents errors related to abnormally low or high patients' weight such as in malnutrition or obesity, or between patients with important racial and/or dietary-related weight differences. The concern was for ARC patients who have a high possibility of subtherapeutic vancomycin levels, even if receiving a daily dose of $3000 \mathrm{mg}$ [8] (presented as $30-45 \mathrm{mg} / \mathrm{kg}$ in [16-18]). The current simulation suggested that daily doses of 3500-4500 mg should be considered for such patients, which is in line with a nomogram previously proposed for critically-ill patients [30]. This may require early identification and separation of ARC patients from the general population, but is feasible as these patients are typically young and with trauma, burn injuries, or acute leukaemia [31,32]. A serum creatinine $<0.4 \mathrm{mg} / \mathrm{dL}$ could also serve as a surrogate marker for ARC during the treatment [17].

The population used for this study is typical of ICU patients who are critically ill, and can therefore be considered as nor- mative. Gender and racial considerations are probably irrelevant because differences in this context primarily relate to drug transport and metabolism and weight. Vancomycin is not significantly metabolised in humans. Its elimination primarily depends on renal function [29,32] (with non-renal clearance being probably $<5 \%$ of the total drug clearance), which the current model and proposed dosing strategies fully consider, and its plasma levels are not influenced by the activity of renal transport systems [33]. Moreover, all current data were adjusted to a standard creatinine clearance of $100 \mathrm{~mL} / \mathrm{min}$ for a nominal 70-kg subject, allowing easy comparison and translation to patients with different renal function and weight.

In conclusion, critically ill patients treated with vancomycin $\mathrm{Cl}$ required higher loading doses than those often recommended for rapidly attaining serum concentrations within a desirable range. However, this range should not be excessively high, to avoid the risk of toxicity, which led to the proposed $20-30 \mathrm{mg} / \mathrm{L}$. The standard daily maintenance dose of $2000-3000 \mathrm{mg}$ is acceptable for most patients with a $\mathrm{CLCr}$ of $61-130 \mathrm{~mL} / \mathrm{min}$ but should definitely be markedly increased for patients with ARC. A global limitation in the current study was the use of the Cockcroft-Gault equation for calculating the CLCr. While more sophisticated equations are available, they did not prove more reliable in daily clinical practice and have not been validated for drug dosage readjustment.

Timeously reaching and steadily maintaining the desired target range will improve the treatment outcome and reduce the burden of TDM performance, which nevertheless remains necessary due to high PK variability between patients and during treatment, since these are critically ill and therefore in unstable conditions and with vital signs not within normal limits.

\section{Acknowledgements}

We thank the General Direction of the Bach Mai Hospital and the Academic Authorities of the Hanoi University of Pharmacy for continuous encouragement in our studies.

Funding support: This work did not receive specific grant from any funding agency in the public, commercial, or not-for-profit sectors, and was covered by the general budget of the participating Institutions. D.H.V. and H.A.N. are staff members of the Vietnamese National Drug Information and Adverse Drug Reaction Monitoring Center, Hanoi University of Pharmacy (HUP), Hanoi, Vietnam; T.T.H and D.A.N are students at HUP; I.K.D. is a post-graduate fellow and supported by the MON4STRAT programme of the European Union (FP7); X.C.D., G.B.N, H.G.D, N.T.T, and H.N.P are staff members of 
the Bach Mai Hospital; F.V.B. is Research Director of the Belgian F.R.S.-FNRS; P.M.T. is an unpaid Emeritus Professor.

\section{Conflicts of interest: Nothing to declare in relation to the work} presented here.

Ethical approval: Ethical approval was obtained from the Ethical Committee of Bach Mai hospital with the reference number of 3327/QD-BM for the protocol BM-2015-728-81.

\section{Registration no. Not applicable (observational study).}

\section{Supplementary materials}

Supplementary material associated with this article can be found, in the online version, at doi:10.1016/j.ijantimicag.2019.09. 018.

\section{References}

[1] Rybak MJ, Lomaestro BM, Rotschafer JC, Moellering RC, Craig WA, Billeter M, et al. Vancomycin therapeutic guidelines: a summary of consensus recommendations from the infectious diseases Society of America, the American Society of Health-System Pharmacists, and the Society of Infectious Diseases Pharmacists. Clin Infect Dis 2009;49:325-7 PMID: 19569969.

[2] Bruniera FR, Ferreira FM, Saviolli LR, Bacci MR, Feder D, da Luz Goncalves Pedreira M, et al. The use of vancomycin with its therapeutic and adverse effects: a review. Eur Rev Med Pharmacol Sci 2015;19:694-700 PMID: 25753888.

[3] Phillips CJ, Wisdom AJ, McKinnon RA, Woodman RJ, Gordon DL. Interventions Targeting the Prescribing and Monitoring of Vancomycin for Hospitalized Patients: A Systematic Review Protocol. Infect Dis Ther 2017;6:557-63 PMID: 29080067.

[4] Holmes NE, Tong SY, Davis JS, van Hal SJ. Treatment of methicillin-resistant Staphylococcus aureus: vancomycin and beyond. Semin Respir Crit Care Med 2015:36:17-30 PMID: 25643268.

[5] Lubenko IY, Strukova EV, Smirnova MV, Vostrov SN, Portnoy YA, Zinner SH, et al. Telavancin and vancomycin pharmacodynamics with Staphylococcus aureus in an in vitro dynamic model. J Antimicrob Chemother 2008;62:1065-9 PMID: 18635520.

[6] Moise-Broder PA, Forrest A, Birmingham MC, Schentag JJ. Pharmacodynamics of vancomycin and other antimicrobials in patients with Staphylococcus aureus lower respiratory tract infections. Clin Pharmacokinet 2004;43:925-42 PMID: 15509186.

[7] Rybak MJ. The pharmacokinetic and pharmacodynamic properties of vancomycin. Clin Infect Dis 2006:42(Suppl 1):S35-9 PMID: 16323118.

[8] Ampe E, Delaere B, Hecq JD, Tulkens PM, Glupczynski Y. Implementation of a protocol for administration of vancomycin by continuous infusion: pharmacokinetic, pharmacodynamic and toxicological aspects. Int J Antimicrob Agents 2013:41:439-46 PMID: 23523733.

[9] Roberts JA, Lipman J. Antibacterial dosing in intensive care: pharmacokinetics, degree of disease and pharmacodynamics of sepsis. Clin Pharmacokinet 2006:45:755-73 PMID: 16884316.

[10] Vazquez-Guillamet C, Kollef MH. Treatment of Gram-positive infections in critically ill patients. BMC Infect Dis 2014;14:92 PMID: 25431211.

[11] Saugel B, Nowack MC, Hapfelmeier A, Umgelter A, Schultheiss C, Thies P, et al. Continuous intravenous administration of vancomycin in medical intensive care unit patients. J Crit Care 2013;28:9-13 PMID: 22459156.

[12] Cataldo MA, Tacconelli E, Grilli E, Pea F, Petrosillo N. Continuous versus intermittent infusion of vancomycin for the treatment of Gram-positive infections: systematic review and meta-analysis. J Antimicrob Chemother 2012;67:17-24 PMID: 22028203.

[13] Wysocki M, Delatour F, Faurisson F, Rauss A, Pean Y, Misset B, et al. Continuous versus intermittent infusion of vancomycin in severe Staphylococcal infections: prospective multicenter randomized study. Antimicrob Agents Chemother 2001;45:2460-7 PMID: 11502515.
[14] Norton K, Ingram PR, Heath CH, Manning L. Risk factors for nephrotoxicity in patients receiving outpatient continuous infusions of vancomycin in an Australian tertiary hospital. J Antimicrob Chemother 2014;69:805-8 PMID: 24107387.

[15] Roberts JA, Taccone FS, Udy AA, Vincent JL, Jacobs F, Lipman J. Vancomycin dosing in critically ill patients: robust methods for improved continuous-infusion regimens. Antimicrob Agents Chemother 2011:55:2704-9 PMID: 21402850.

[16] Cristallini S, Hites M, Kabtouri H, Roberts JA, Beumier M, Cotton F, et al. New Regimen for Continuous Infusion of Vancomycin in Critically Ill Patients. Antimicrob Agents Chemother 2016;60:4750-6 PMID: 27216073.

[17] Ocampos-Martinez E, Penaccini L, Scolletta S, Abdelhadii A, Devigili A, Cianferoni S, et al. Determinants of early inadequate vancomycin concentrations during continuous infusion in septic patients. Int J Antimicrob Agents 2012;39:332-7 PMID: 22333933.

[18] Baptista JP, Sousa E, Martins PJ, Pimentel JM. Augmented renal clearance in septic patients and implications for vancomycin optimisation. Int J Antimicrob Agents 2012;39:420-3 PMID: 22386742.

[19] Jonsson EN, Karlsson MO. Xpose-an S-PLUS based population pharmacokinetic/pharmacodynamic model building aid for NONMEM. Comput Methods Programs Biomed 1999;58:51-64 PMID: 10195646.

[20] Lindbom L, Pihlgren P, Jonsson EN. PsN-Toolkit-a collection of computer intensive statistical methods for non-linear mixed effect modeling using NONMEM Comput Methods Programs Biomed 2005;79:241-57 PMID: 16023764

[21] Holford NH. A size standard for pharmacokinetics. Clin Pharmacokinet 1996;30:329-32 PMID: 8743333.

[22] Anderson BJ, Holford NH. Mechanism-based concepts of size and maturity in pharmacokinetics. Annu Rev Pharmacol Toxicol 2008:48:303-32 PMID: 17914927.

[23] Allegaert K, Anderson BJ, Cossey V, Holford NH. Limited predictability of amikacin clearance in extreme premature neonates at birth. $\mathrm{Br}$ J Clin Pharmacol 2006;61:39-48 PMID: 16390350.

[24] Hooker AC, Staatz CE, Karlsson MO. Conditional weighted residuals (CWRES) a model diagnostic for the FOCE method. Pharm Res 2007;24:2187-97 PMID: 17612795.

[25] Karlsson MO, Savic RM. Diagnosing model diagnostics. Clin Pharmacol Ther 2007;82:17-20 PMID: 17571070.

[26] Garnacho-Montero J, Garcia-Garmendia JL, Barrero-Almodovar A Jimenez-Jimenez FJ, Perez-Paredes C, Ortiz-Leyba C. Impact of adequate empirical antibiotic therapy on the outcome of patients admitted to the intensive care unit with sepsis. Crit Care Med 2003;31:2742-51 PMID: 14668610.

[27] Martirosov DM, Bidell MR, Pai MP, Scheetz MH, Rosenkranz SL, Faragon C, et al. Relationship between day 1 and day 2 Vancomycin area under the curve values and emergence of heterogeneous Vancomycin-intermediate Staphylococcus aureus (hVISA) by Etest(R) macromethod among patients with MRSA bloodstream infections: a pilot study. BMC Infect Dis 2017;17:534 PMID: 28764660.

[28] Medellin-Garibay SE, Romano-Moreno S, Tejedor-Prado P, Rubio-Alvaro N, Rueda-Naharro A, Blasco-Navalpotro MA, et al. Influence of Mechanical Ventilation on the Pharmacokinetics of Vancomycin Administered by Continuous Infusion in Critically Ill Patients. Antimicrob Agents Chemother 2017;61 PMID: 28893792.

[29] Moellering RC Jr, Krogstad DJ, Greenblatt DJ. Pharmacokinetics of vancomycin in normal subjects and in patients with reduced renal function. Rev Infect Dis 1981(3 suppl):S230-5 PMID: 7342286.

[30] Jeurissen A, Sluyts I, Rutsaert R. A higher dose of vancomycin in continuous infusion is needed in critically ill patients. Int J Antimicrob Agents 2011;37:75-7 PMID: 21074374

[31] Udy AA, Roberts JA, Boots RJ, Paterson DL, Lipman J. Augmented renal clearance: implications for antibacterial dosing in the critically ill. Clin Pharmacokinet 2010;49:1-16 PMID: 20000886.

[32] Cook AM, Hatton-Kolpek J. Augmented Renal Clearance. Pharmacotherapy 2019;39:346-54 PMID: 30723936.

[33] Wen S, Wang C, Huo X, Meng Q, Liu Z, Yang S, et al. JBP485 attenuates vancomycin-induced nephrotoxicity by regulating the expressions of organic anion transporter (Oat) 1, Oat3, organic cation transporter 2 (Oct2), multidrug resistance-associated protein 2 (Mrp2) and P-glycoprotein (P-gp) in rats. Toxicol Lett 2018;295:195-204 PMID: 29964132. 
Vu et al.

Determination of optimal loading and maintenance doses for continuous infusion of vancomycin in critically ill patients: population pharmacokinetic modelling and simulations for improved dosing schemes.

\section{Supplementary Material}

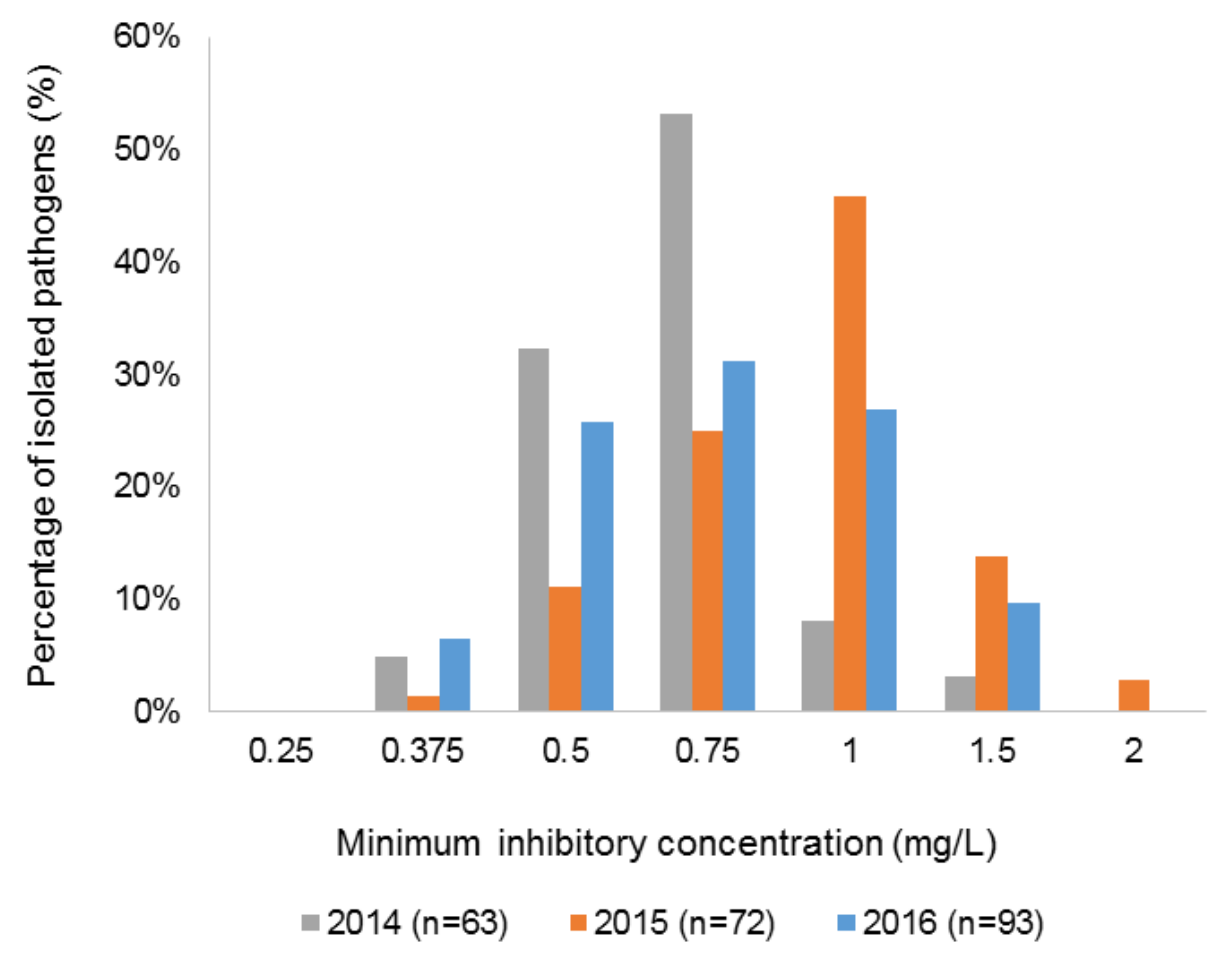

Figure S1. Distribution of minimum inhibitory concentrations of vancomycin against Staphylococcus aureus strains isolated from patients during 3 consecutive years at the Bach Mai hospital, Hanoi, Vietnam. 